ARTICLE

\title{
Understanding and responding to HIV risk in young South African women: Clinical perspectives
}

\author{
R Dellar, ${ }^{1,2}$ MBiochem Molecular and Cellular Biochemistry (Oxon); A Waxman, ${ }^{1}$ MSc Public Health in Developing Countries; \\ Q Abdool Karim, ${ }^{1,3} \mathrm{PhD}$ (Medicine) \\ ${ }^{1}$ Centre for the AIDS Programme of Research in South Africa (CAPRISA), University of KwaZulu-Natal, Durban, South Africa \\ ${ }^{2}$ Africa Centre for Health and Population Studies, University of KwaZulu-Natal, Durban, South Africa \\ ${ }^{3}$ Mailman School of Public Health, Columbia University, New York, USA
}

Corresponding author: R Dellar (rachael.dellar@gmail.com)

Young women (15 - 24 years) contribute a disproportionate 24\% to all new HIV infections in South Africa - more than four times that of their male peers. HIV risk in young women is driven by amplifying cycles of social, behavioural and biological vulnerability. Those most likely to acquire infection are typically from socioeconomically deprived households in high HIV-prevalence communities, have limited or no schooling, engage in transactional sex or other high-risk coping behaviours, and have a history of sexually transmitted infections (STIs) and/or pregnancy. Despite the imperative to prevent HIV acquisition in young women, there is a dearth of evidence-based interventions to do so. However, there are several steps that healthcare workers can take to improve outcomes for this key population at the individual level. These include being able to identify high HIV-risk young women, ensuring that they receive the maximum social support they are eligible for, providing reliable and non-judgemental counselling on sexual and reproductive health and relationships, delivering contraceptives and screening and treating STIs in the context of accessible, youth-friendly services.

S Afr Med J 2015;105(11):952. DOI:10.7196/SAMJ.2015.v105i11.10099

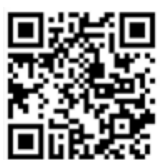

Despite huge improvements in access to antiretroviral therapy (ART) and an overall decline in HIV incidence in South Africa (SA), young women (15 - 24 years) remain uniquely vulnerable to infection. They contribute about a quarter of all new HIV infections occurring in SA and are thus key to epidemic control. Understanding and responding to the risk they face is a public health imperative.

Many studies have sought to characterise factors associated with higher vulnerability to HIV in young women. Together, these studies paint a picture of an amplifying cycle of risk for many young South Africans. Typically, this cycle is founded in poor socioeconomic backgrounds that drive engagement in high-risk sexual behaviours and expose young women to risks of sexual abuse, sexually transmitted infections (STIs) and pregnancy. Directly and indirectly, all these experiences have substantial implications for the odds of young women acquiring HIV. Those at greatest risk can be identified and prioritised by healthcare workers as being from unstable or child-headed households, not being in school, engaging in transactional sex, being victims of gender-based violence and/or having a history of pregnancy or STIs.

Despite the current lack of biological technologies available to reduce HIV acquisition risk in young women, healthcare workers may have a substantive impact on young women's lives at the individual level by helping them to break out of the cycles of vulnerability they face. Ensuring that young people receive the full available social and financial support they are eligible for via governmental and non-governmental organisations is an important first step, especially considering the many structural factors that drive high-risk behaviours in this key population. Healthcare workers can also provide reliable information on sexual and reproductive health. Importantly, such counselling should be tailored to the individual recipient and aim to include discussions about healthy relationships and female genital cleaning practices in addition to the more standard risk-reduction curriculum. Providing access to STI screening, STI treatment, and family planning services is key.

Critical to all these healthcare worker-initiated strategies is an environment in which young people feel comfortable: a nonjudgemental, non-stigmatising, confidential service. Clinics in high HIV-prevalence areas might consider developing specially trained youth-friendly teams that serve clinics at specific hours suited to young people. Furthermore, in rural communities, mobile treatment and education services could reach more isolated young women.

Healthcare worker cognizance of the real-life context of the lives of young women is vital to assisting them in reducing their HIV risk and supporting the public health goals of epidemic control.

\section{Why is it important to address HIV risk in SA's young women?}

Preventing new HIV infections in adolescent girls and young SA women is a public health imperative. This key population has an unprecedentedly high risk of acquiring HIV. In 2012, it was estimated that 1 young SA woman (15 - 24 years) was infected every 5 minutes. ${ }^{[1]}$ Further, given that a quarter of all new infections in SA occur in young women, the goals of an 'AIDS-free generation' will not be achieved without reducing their risk of HIV acquisition. ${ }^{[1]}$

\section{What do we know about what contributes to HIV risk in young people?}

A large number of studies have sought to characterise social, behavioural and biological factors associated with HIV risk in young women (Box 1). The factors fall into several broad categories that describe a network of inter-related risk (Fig. 1).

At a structural level, young women at greatest risk of HIV acquisition are those from socioeconomically deprived communities 


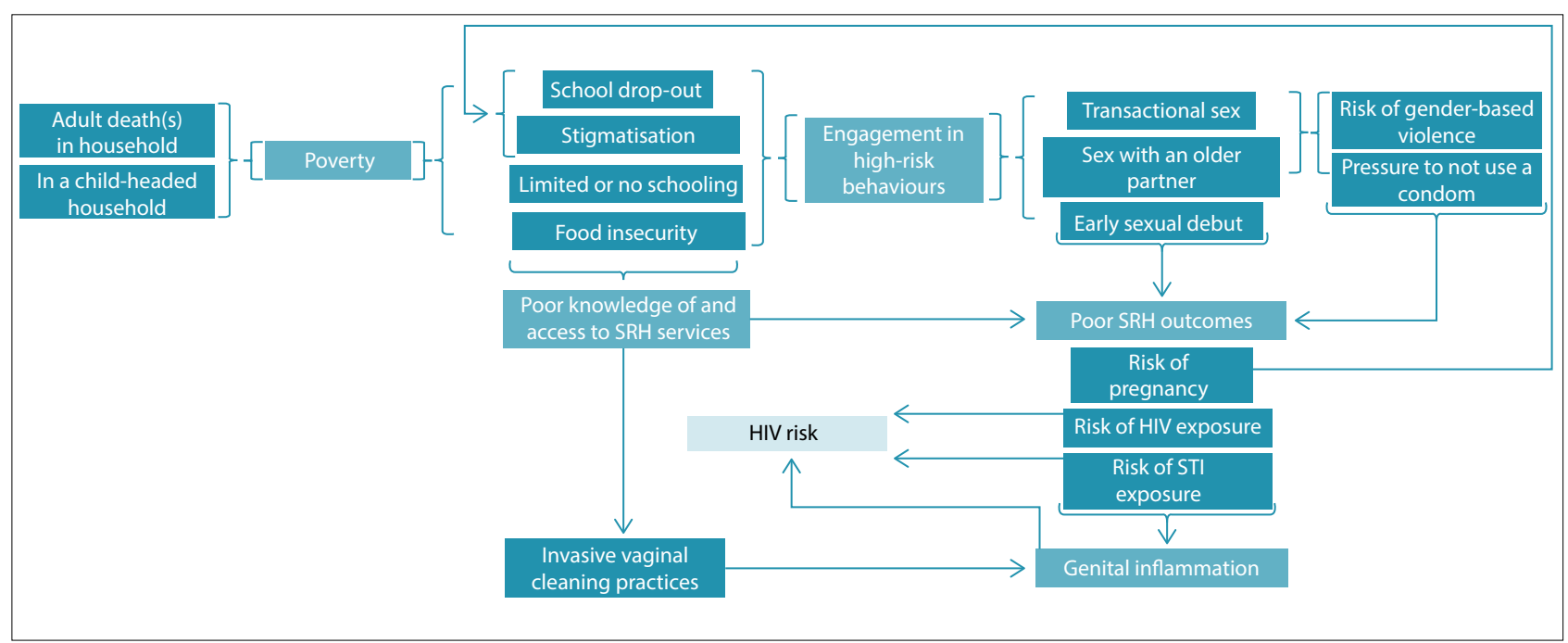

Fig. 1. Network of amplifying risk for young South African women (SRH = sexual and reproductive health).

\section{Box 1. Factors associated with being HIV-positive and/or with HIV acquisition}

Factors associated with HIV risk in young people from southern Africa have previously been reviewed ${ }^{[2]}$ and include: living in a high HIV-prevalence area with low ART coverage; being from a household with poor socioeconomic indicators, including food insecurity and adult death; not being in school or having few years of schooling; a relatively early sexual debut; more than the average number of lifetime sexual partners; sex with an older partner, potentially in a transactional relationship (sex for gifts or favours); inconsistent condom use; a history of sexually transmitted infections; and a history of pregnancy.

Most of these factors have been identified via cross-sectional studies and thus show associations with being HIV-positive. Only a few factors have been tested and confirmed in longitudinal studies that follow a cohort over time as being associated with higher HIV acquisition risk.

with high levels of unemployment, high background HIV prevalence, and poor access to ART programmes. ${ }^{[1,3]}$ In these communities, the most vulnerable households include those with histories of premature adult deaths (often AIDS related), typically with a degree of food insecurity. Young women from child-headed households are at particular risk (Box 2: Lihle's story). ${ }^{[4]}$

Young women from poor socioeconomic backgrounds are more likely to have few years of schooling, leading to poor knowledge of sexual and reproductive processes and services available to them (Box 2: Thembe's story). ${ }^{[4]}$ Furthermore, young women living in such settings may also engage in high-risk behaviours to either relieve immediate survival challenges or because of a limited sense of a potential better future. ${ }^{[5,6]}$ These behaviours may include early sexual debut and engagement in transactional sex (sex for gifts or favours, rather than formal prostitution), often with older partners with higher HIV prevalence than their peers (Box 2: Sarah's story). ${ }^{[7,8]}$ Young women experiencing gender-based violence are also at high risk of HIV acquisition, as negotiating condom use in such relationships is especially difficult. ${ }^{[9]}$

If engagement in high-risk behaviours does not directly result in HIV infection, it often leads to other poor sexual and reproductive

\section{Box 2. Case studies of HIV risk from rural KwaZulu-Natal}

Three stories compiled from a qualitative study on the lives of young women in a particularly high HIV-prevalence rural district of KwaZulu-Natal highlight the real-life context of HIV risk in SA: ${ }^{[12]}$

- Lihle's story. Lihle is 17 years old and, following the death of her mother from AIDS, is living in a child-headed home. She does not attend school because she is looking after the home and her younger siblings. Because of her family's state of poverty, she engages in transactional sex with older men. She says that 'men give you something because they want to have sex with you ... I think they use your financial situation to their benefit, to satisfy their need'. She is worried about getting HIV and about her personal safety, 'sometimes I have sex expecting nothing in return, sometimes I am coerced and expect any outcome.

- Thembe's story. Thembe is a 19-year-old girl from the local rural area. Her boyfriend lives in another town. She has unprotected sex with him once or twice a month when she visits him. Although she wants to use a condom, he repeatedly refuses. He says that he wants to have children with her, even though he is currently expecting a child with another young woman. She doesn't want to have children and is worried about contracting HIV, but he tells her that 'having unprotected sex makes your partner love you more'. She considered using the contraceptive medroxyprogesterone (Depo-Provera) to prevent pregnancy, but she has heard that 'at some point your womb is closed [if you don't conceive when you are young]' and she is concerned because she 'doesn't know where your blood goes when you don't menstruate'.

- Sarah's story. Sarah is 16 years old and is already HIVpositive. She dropped out of school earlier in the year following an unplanned pregnancy. She says she was tricked into pregnancy by her abusive boyfriend, who once 'sweet and innocent' is now 'too clever'. She would like him to 'stop hitting and cheating with other girls'. As a result of her pregnancy and school drop-out, Sarah became stigmatised by her community. She has defaulted on her HIV treatment because she doesn't like to leave the house, and there is no one available to ensure that she takes it regularly. She has recently been diagnosed with TB. 
health outcomes that compound risk. Herpes simplex virus 2 (HSV-2), human papillomavirus (HPV) and other STIs can all leave women biologically more susceptible to HIV for every exposure they have. ${ }^{[10]}$ Certain female genital cleansing practices (including douching and insertion of foreign substances) may also mediate risk via inflammation. ${ }^{[11]}$ Moreover, engaging in unprotected sex carries a risk of pregnancy, which can lead to stigmatisation and school drop-out, and may amplify the risks of gender-based violence or engagement in transactional sex owing to vulnerability and material need, respectively (Box 2: Sarah's story).

\section{What tools do healthcare workers have to reduce the risk of young people becoming infected with HIV?}

Given the current lack of availability of effective biomedical HIV prevention technologies ${ }^{[2,12]}$ and the importance of structural factors in driving HIV in young SA women, it might seem that there is not much healthcare workers can do to support the former in reducing their odds of acquiring HIV, except advocate for broader economic and scientific development (Box 3). However, a closer look at the case studies in Box 2 reveals a number of ways in which healthcare workers might intervene to break young women out of the cycles of amplifying risk they face.

The first action in helping to reduce the vulnerability of their young female patients is confirming that those in severe financial need, or from child-headed households, receive the full governmental support (both financial and social) available to them. In some cases, referral to local non-governmental organisations for additional aid may also be an option. Ensuring that young women such as Lihle receive adequate support is essential in preventing them feeling forced into engaging in transactional sex or other high-risk behaviours for immediate survival.

A second action that healthcare workers can take is to provide reliable and non-judgemental counselling on sexual and reproductive health. Although such counselling is usually available, lack of healthcare worker expertise or time often means

Box 3. Future tools for HIV prevention in young SA women Despite the risk faced by young SA women, there is a dearth of evidence-based HIV prevention interventions available to them. ${ }^{[2,12]}$ No biomedical technologies to prevent HIV are yet available, and although school- and community-based education programmes are common, few have been evaluated for efficacy. Two promising future tools in HIV prevention in young women are pre-exposure prophylaxis (PrEP) and conditional cash transfers.

- PrEP: PrEP is the administration of antiretrovirals in HIVnegative individuals for the prevention of HIV infection. Many variations of PrEP have been or are being developed, including oral, topical, barrier and injectable formulations. Despite early trials demonstrating the effectiveness of PrEP for HIV prevention, implementation has been slowed by problems with adherence highlighted in more recent studies. ${ }^{[15]}$

- Conditional cash transfers: Cash transfers that incentivise behaviours thought to reduce HIV risk (including promoting remaining in school) have recently emerged as a promising new strategy to reduce young people's vulnerability to $\mathrm{HIV}^{[2,16]}$ Two randomised controlled trials undertaken in SA (HPTN 068 and CAPRISA 007) were unable to demonstrate any impact on HIV acquisition as a result of lower than anticipated HIV incidence rates. that key risk factors are not adequately addressed. For example, if Thembe was given complete information on fertility control, she might have chosen to initiate hormonal contraceptives, thereby reducing her pregnancy risk, avoiding dropping out of school, and ultimately reducing her HIV risk. Counselling with regard to condom use should be included, but healthcare workers should be aware that young women often have limited say in this decision. If couple counselling or engaging with a male partner is not possible, counselling should extend to relationships and negotiating advice on safer sexual matters. Another important extension of counselling that is currently rarely performed is the discussion of inappropriate vaginal cleaning practices that may increase susceptibility to HIV.

A third intervention point in the cycles of HIV risk faced by young women is the treatment of STIs other than HIV. Treating HSV-2 and other STIs is critical to ensure that young people's HIV exposure risk is reduced. Therefore, all young people attending clinics should be screened for STIs, even if this is not the primary purpose of their visit.

Prerequisites to all of these interventions is the provision of accessible services prioritised for the most HIV-vulnerable young women. All healthcare workers should be able to identify young people at highest HIV risk (using the above associations as guiding points) and have an obligation to ensure that they are providing non-stigmatising and completely confidential services. Furthermore, clinics in high-risk areas might consider the additional provision of mobile youth-friendly sexual and reproductive health education programmes and services to reach those without access to or not utilising fixed healthcare facilities. ${ }^{[14]}$

\section{Conclusions}

Understanding and responding to the unprecedented levels of HIV risk in young SA women is a public health imperative for continued progress towards control of the HIV epidemic. Healthcare workers have a vital role to play in reducing this risk, as they have the opportunity to make substantial changes to young women's lives at the individual level. Maximising this benefit requires healthcare workers to be able to understand the complexity of what drives vulnerability to HIV in young women so that they can identify women who require special attention, such as those from childheaded households, those not in school, and those engaging in transactional sex. Understanding the drivers of HIV is also critical in developing appropriate intervention strategies to break down cycles of risk. At the core of these healthcare worker-initiated intervention strategies is ensuring that vulnerable young people have access to the social support available to them, receive reliable, extensive and non-judgemental counselling, and are regularly screened for STIs. Developing youth-friendly services tailored to meet the unique sexual and reproductive health needs of young people is also critical.

Acknowledgements. Rachael Dellar was previously supported by the CAPRISA Fellowship Training Programme and is currently funded by an MRC Flagship Grant (MRC-RFA-UFSP-01-2013/UKZN HIVEPI) Aliza Waxman was a CAPRISA trainee supported by a Fogarty Fullbright Scholarship. The case studies in Box 2 were compiled from a series of interviews with young women, undertaken as part of a larger qualitative research study (approved by the University of KwaZulu-Natal (UKZN) Humanities and Social Sciences Research Ethics Committee (HSS REC/0019/014)). 


\section{References}

1. Shisana O, Rehle T, Simbayi LC. South African National HIV Prevalence Incidence and Behaviour Survey 2012. Cape Town: HSRC Press, 2014

2. Dellar RC, Dlamini S, Karim QA. Adolescent girls and young women: Key populations for HIV epidemic control. J Int AIDS Soc 2015;18(2 Suppl 1):19408. [http://dx.doi.org/10.7448/ ias.18.2.19408]

3. Pettifor AE, Rees HV, Kleinschmidt I, et al. Young people's sexual health in South Africa: HIV prevalence and sexual behaviors from a nationally representative household survey. AIDS 2005;19(14):1525-1534. 4. Hallman K. Gendered socioeconomic conditions and HIV risk behaviours among young people in South Africa. Afr J AIDS Res 2005;4:37-50. [http://dx.doi.org/10.2989/16085900509490340]

5. Chatterii M, Murray N, London D, Anglewicz P. The factors influencing transactional sex among young men and women in 12 sub-Saharan African countries. Soc Biol 2005;52(1-2):56-72. http:// young men and women in 12 sub-Saharan
dx.doi.org/10.1080/19485565.2002.9989099 6. Weiser SD, Leiter K, Bangsberg DR, et al. Food insufficiency is associated with high-risk sexual
behavior among women in Botswana and Swaziland. PLoS Med 2007;4(10):1589-1597; discussion behavior among women in Botswana and Swaziland. PLo
1598. [http://dx.doi.org/10.1371/journal.pmed.0040260]

1598. [http://dx.doi.org/10.1371/journal.pmed.0040260]
7. Dunkle KL, Jewkes RK, Brown HC, Gray GE, McIntryre JA, Harlow SD. Transactional sex amon women in Soweto South Africa: Prevalence risk factors and association with HIV infection. Soc Sci Med 2004;59(8):1581-1592. [http://dx.doi.org/10.1016/j.socscimed.2004.02.003]

8. Stoebenau K, Nixon SA, Rubincam C, et al. More than just talk: The framing of transactional sex and its implications for vulnerability to HIV in Lesotho, Madagascar and South Africa. Global Health 2011;7:34. [http://dx.doi.org/10.1186/1744-8603-7-34]

9. Pettifor AE, Measham DM, Rees HV, Padian NS. Sexual power and HIV risk South Africa. Emerg Infect Dis 2004;10(11):1996-2004. [http://dx.doi.org/10.3201/eid1011.040252]

10. Cohen MS. HIV and sexually transmitted diseases: Lethal synergy. Top HIV Med 2004;12(4):104-107.
11. McClelland RS, Lavreys L, Hassan WM, Mandaliya K, Ndinya-Achola JO, Baeten JM. Vaginal washing and increased risk of HIV-1 acquisition among African women: A 10-year prospective study. AIDS 2006;20(2):269-273. [http://dx.doi.org/10.1097/01.aids.0000196165.48518.7b]

12. Waxman A, Humphries $\mathrm{H}$, Frohlich J, et al. Young women's life experiences and perceptions of sexual and reproductive health in rural KwaZulu-Natal South Africa (in preparation).

13. Mavedzenge SN, Luecke E, Ross DA. Effectiveness of HIV Prevention Treatment and Care Interventions Among Adolescents: A Systematic Review of Systematic Reviews. UNICEF Technical Brief. New York: UNICEF, 2013.

14. Denno DM, Chandra-Mouli V, Osman M. Reaching youth with out-of-facility HIV and reproductive health services: A systematic review. J Adolesc Health 2012;51(2):106-121. [http://dx.doi.org/10.1016/j. jadohealth.2012.01.004]

15. Bekker L-G, Gill K, Wallace M. Pre-exposure prophylaxis for South African adolescents: What evidence? S Afr Med J 2015;105(11):907-911. [http://dx.doi.org/10.7196/SAMJ.2015.v105i11.10222]

16. Pettifor A, MacPhail C, Nguyen N, Rosenberg M. Can money prevent the spread of HIV? A review of cash payments for HIV prevention. AIDS Behav 2012;16(7):1729-1738. http://dx.doi.org/10.1007/ s10461-012-0240-z

\section{Continuing medical education resources}

Dellar RC, Dlamini S, Abdool Karim Q. Adolescent girls and young women: Key populations for HIV epidemic control. J Int AIDS Soc 2015;8(2 Suppl 1):19408.

Shisana O, Rehle T, Simbayi LC, et al. South African National HIV Prevalence, Incidence and Behaviour Survey, 2012. Cape Town: HSRC Press, 2014. http://www.hsrc.ac.za/uploads/pageContent/4565/ SABSSM\%20IV\%20LEO\%20final.pdf (accessed 23 September 2015).

UNICEF Technical Brief. Effectiveness of HIV Prevention, Treatment and Care Interventions Among Adolescents: A Systematic Review of Systematic Reviews. UNICEF: New York, 2013. 